\title{
Apreciación del docente para contribuir al desarrollo del pensamiento crítico*
}

\section{Assessment of teachers to contribute to the development of critical thinking}

\author{
María Patricia Gómez-GÓmez** \\ Sandra Milena Botero-Bedoya***
}

\begin{abstract}
Resumen
Objetivo. Determinar los métodos de enseñanza y estrategias educativas que contribuyen al desarrollo del pensamiento crítico. Metodología. La siguiente investigación se realizó bajo un enfoque cualitativo, de paradigma interpretativo y un método inductivo, que proporcionan al lector una puesta al día sobre conceptos útiles en áreas en constante evolución. Participaron 14 docentes adscritos al programa de la Licenciatura en Educación Infantil a quienes de manera individual se les realizó una entrevista semiestructurada. Resultados. Se encontró que el desarrollo del pensamiento crítico en el aula por medio de diferentes métodos y estrategias que emplea el docente favorecen habilidades y capacidades cognitivas que promueven en el estudiante la capacidad de organizar, sistematizar las ideas y sintetizar criterios elementales, de manera razonable, reflexiva y coherente. Conclusión. Favorecer el desarrollo del pensamiento crítico en la educación superior debe ser un objetivo transversal de todos los currículos de formación.
\end{abstract}

Palabras clave: desarrollo de pensamiento, docentes, estrategias educativas, método de enseñanza.

\section{Abstract}

Objective:To determine teaching methods and educational strategies that contribute to the development of critical thinking. Methodology: This research was carried out under a qualitative approach, the interpretive paradigm and an inductive method which provides the reader with an update on useful concepts in constantly evolving areas. Fourteen teachers of the Bachelor's Degree Program in Early Childhood Education, who were individually interviewed in a semi-structured interview, participated in the program. Results: It was found that the development of critical thinking in the classroom through different methods and strategies used by the teacher, favor cognitive abilities and capacities that promote in the students the ability to organize, systematize ideas and synthesize elementary criteria in a reasonable, reflective and coherent manner. Conclusion: Promoting the development of critical thinking in higher education should be a cross-cutting objective of all training curricula.

Key words: development of thought, teachers, educational strategies, teaching method.

\footnotetext{
* La presente investigación hace parte del artículo de revisión "Pensamiento crítico y su relación con las experiencias áulicas de las estudiantes de la Licenciatura Educación Infantil en la Universidad Católica Luis Amigó”.

*** Universidad Católica Luis Amigó. Medellín, Colombia. E-mail: maria.gomezgo@amigo.edu.co.

(D) orcid.org/0000-0002-6456-8813 Google Scholar

*** Universidad Católica Luis Amigó. Medellín, Colombia. E-mail: sandra.boterobe@amigo.edu.co.

(D) orcid.org/0000-0002-5415-729 Google Scholar
} 


\section{Introducción}

El desarrollo del pensamiento crítico en los estudiantes universitarios ha sido una necesidad latente en la educación, situación reflejada en los resultados de las pruebas Saber Pro, y que develan los conocimientos plenos alcanzados por los estudiantes en su formación; además, propician con ello la confrontación de sus habilidades, competencias disciplinares y actitudinales, las cuales han desarrollado en diferentes contextos y situaciones vivenciales como sujetos con pensamiento reflexivo y participativo en la sociedad. Romero, Villareal, Samper y Ospino (2017) exponen que "las universidades de Colombia (...) por medio del Ministerio de Educación Nacional (MEN), establecen los parámetros de medición de las diferentes competencias, de acuerdo con las carreras profesionales" (p. 3).

Adicionalmente, y de acuerdo con los resultados arrojados por el Programa para la Evaluación Internacional de Alumnos (PISA) en sus versiones 2006, 2009 y 2012 para la competencia de lectura crítica, estableció que los estudiantes colombianos para esos años estaban por debajo del nivel básico. Sin embargo, en la medición del 2015 se evidencia el progreso de Colombia en esta competencia (gráfica 1).

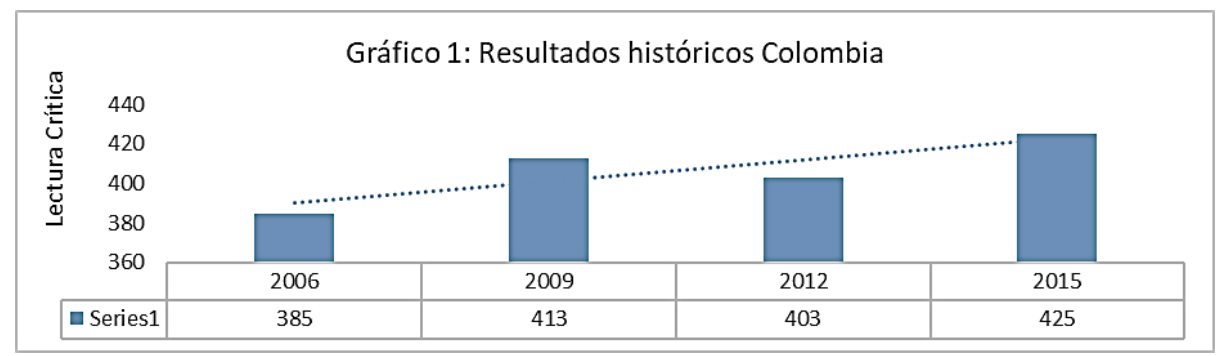

Gráfico 1. Resultados históricos Colombia.

Fuente: elaboración propia.

Con respecto al gráfico 1, los criterios de competencia del pensamiento crítico en Colombia se mantienen sobre la media, por lo que se debe seguir trabajando para alcanzar resultados que nos posesionen sobre niveles de orden superior, que den cuenta del trabajo interdisciplinar realizado desde los diferentes componentes educativos, que aporte a disminuir la brecha frente a países como Chile y Uruguay que tienen los desempeños más altos de la región. En este sentido, y de acuerdo con las estadísticas presentadas por PISA (2015) a nivel de país, Chile ocupa el primer lugar de la región, en el lugar 44, seguido de Uruguay (47), Costa Rica (55), Colombia (57), México (58), Brasil (63), Perú (64) y República Dominicana (70). 
En este orden de ideas se comprende la importancia del desarrollo del pensamiento crítico en la educación. Sobre este tema se puede referenciar el estudio realizado en el contexto nacional por Steffens et al. (2017), quienes concluyen que los resultados arrojados en las pruebas Saber Pro han develado que los universitarios colombianos, pese a la ubicación por encima o igual del promedio estándar nacional, demuestran bajos niveles en el desarrollo del pensamiento crítico; incluso, algunos de estos estudiantes terminan su formación profesional sin alcanzar los niveles adecuados.

Paul y Elder (2005) definen el concepto "pensamiento crítico" como "el proceso de analizar y evaluar el pensamiento con el propósito de mejorarlo. El pensamiento crítico presupone el conocimiento de las estructuras más básicas del pensamiento” (p. 7). Franco, Piedra y Cunalema (2018) lo plantean como una modalidad de razonar que moviliza el acto de entender y establecer una organización sistémica de las ideas, procesos que promueven en el estudiante habilidades y capacidades cognitivas de análisis, indagación, reflexión, investigación, conceptualización y verificación de datos. El proceso de aprendizaje, en lo que respecta al desarrollo del pensamiento crítico, está relacionado con las estrategias educativas y métodos de enseñanza que implementan los docentes: "La enseñanza de las habilidades requiere de la figura del profesor como guía, que lleve al estudiante, gradualmente, a mayores competencias $\mathrm{y}$, a su vez, le permita asumir el control del proceso de la actividad cognitiva y meta-cognitiva" (Mateos como se citó en Osses y Jaramillo, 2008, p. 92).

Ortiz (como se citó en Montes de Oca y Machado, 2011) utiliza la expresión estrategia educativa, "lo cual presupone enfocar el cómo enseña el docente y cómo aprende el alumno a través de un proceso donde los últimos aprenden a pensar y a participar activa, reflexiva y creadoramente" (p. 8). Lo anterior permite relacionar las estrategias educativas con los métodos de enseñanza en los procesos formativos de enseñanza-aprendizaje para que, utilizando el contenido, los estudiantes alcancen el objetivo.

\section{Metodología}

En lo concerniente a la metodología, el tipo de investigación que se realizó fue a través del enfoque cualitativo, de paradigma interpretativo y el método inductivo. Teniendo en cuenta que el enfoque cualitativo suministra al lector información sobre conceptos valiosos en áreas en permanente evolución. De acuerdo con Sampieri (2004), se trata de buscar información importante para examinar hechos o acontecimientos.

Paradigma interpretativo: evidencia una relación de participación democrática y comunicativa entre el investigador y el objeto investigado. En consonancia con Martínez (2011), "existen múltiples realidades construidas por los actores en su relación con la realidad social en la cual viven. Por eso, no existe una sola verdad, sino que surge como una configuración de los diversos significados que las personas le dan (...)” (p. 9). 


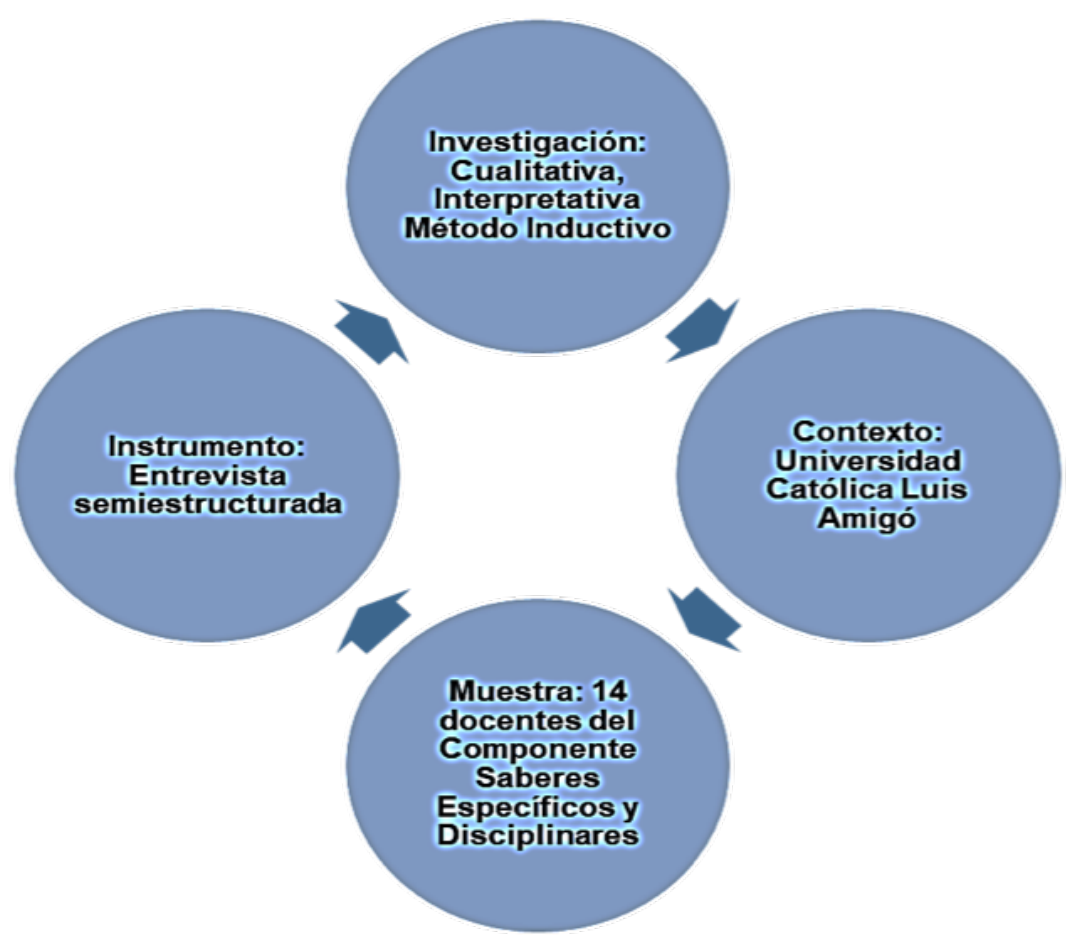

Gráfica 2. Metodología.

Fuente: elaboración propia.

Método inductivo: el estudio que se realizó parte de pautas de los datos que se consideraron pertinentes en el diseño de la entrevista semiestructurada para definir los métodos y estrategias educativas, relacionando cada método como la forma o procedimiento para diseñar las estrategias, en contribución al desarrollo del pensamiento crítico. El método dialógico es el más utilizado por los docentes ya que es consecuente para favorecer la adquisición de competencias referidas al desarrollo del pensamiento crítico, la argumentación, la comunicación no verbal, la capacidad crítica y autocrítica, las relaciones interpersonales y el manejo de frustraciones y sentimientos, generando en los estudiantes competencias tales como actitudes, conocimientos, capacidades y habilidades en tanto la periodicidad de confluencia entre estudiantes y docentes. También permite encuentros de conocimientos, sentires, emociones, intereses, necesidades, sueños y expectativas, a partir de cuya comprensión se establecen horizontes comunes que guían el proceso educativo.

Técnica e instrumentos: con el fin de determinar los métodos de enseñanza y estrategias educativas que contribuyen al desarrollo del pensamiento crítico se diseñó y aplicó en agosto y septiembre del 2019 una entrevista semiestructurada que requirió de un guion de entrevista; 
no obstante, las cuestiones se elaboraron de forma abierta y se estructuró con un total de ocho preguntas, planteadas a 14 docentes adscritos al programa de la Licenciatura en Educación Infantil. Luego se analizó a través de una matriz categorial, cada entrevista se tabuló de manera independiente y a partir de ello se clasificaron y estudiaron todos los datos recolectados; de esos datos se obtuvo la hipótesis de fortalecer en espacios académicos la formación en la competencia de análisis crítico y la contribución al conocimiento científico, reconociendo la capacidad autónoma de los estudiantes en cuanto a la formulación de preguntas, proyectos y resolución de problemas. Lo anterior, en relación al pensamiento crítico, las estrategias y métodos de enseñanza que permitieron su lectura e interpretación y es, además, resultado de la comparación entre dichas categorías.

Participantes: la muestra estuvo conformada por 14 docentes de la Licenciatura en Educación Infantil, los criterios de selección que se tuvieron en cuenta fueron: ser docente del componente saberes específicos y disciplinares, contar con un mínimo de dos años en la universidad, tener un contrato de tiempo completo, conocer las necesidades y realidades de formación de maestros para la infancia en el contexto actual a partir de políticas, principios, valores y acciones que se consolidan en la misión institucional: "La Universidad Católica Luis Amigó es una Institución de Educación Superior (...) para divulgar el conocimiento científico, tecnológico y cultural y para la formación de profesionales con conciencia crítica, ética y social”.

Herramienta: la entrevista semiestructurada fue avalada por tres expertos en educación superior y el departamento de Investigación de la Universidad Católica Luis Amigó, constatando la importancia de determinar los métodos de enseñanza y estrategias educativas que contribuyen al desarrollo del pensamiento crítico; en este sentido "los maestros tienen la responsabilidad de generar espacios en las escuelas que les permitan a sus estudiantes desarrollar estrategias para comunicarse asertivamente y fomentar su pensamiento crítico" (Chaux como se citó en Palacio, Rodríguez y Gallego, 2019, p. 40). La estructura de la entrevista se diseñó en concordancia con el objeto de estudio a interpretar y tiene relación directa con las categorías de la investigación; asimismo, el entrevistado hizo lectura del consentimiento informado que detalla con claridad cada uno de los parámetros de confidencialidad.

Procedimiento: la entrevista semiestructurada fue elaborada por los investigadores de esta propuesta e integró ocho preguntas abiertas. Cada una de ellas respondió de manera coherente a las categorías de la investigación: desarrollo del pensamiento crítico, estrategias educativas y métodos de enseñanza. El proceso se llevó a cabo de la siguiente manera: 1. Organización de la aplicación de la entrevista, 2. Se definió la cantidad de docentes por el campo de formación, 3. Se acordó disponibilidad de horarios para realizar la entrevista. Estas se desarrollaron individualmente dando una breve explicación de la finalidad del estudio, sin contar con un tiempo límite para este proceso. 
Apreciación del docente para contribuir al desarrollo del pensamiento crítico

Se elaboró una matriz para dar cuenta de la triangulación de la información, es decir, contrastarla con la teoría para comprender la importancia del tema en la Universidad Católica Luis Amigó y, al mismo tiempo, la importancia de las estrategias educativas implementadas en los diferentes cursos. Acerca del método de triangulación, Bisquerra (2004) expresa que es un proceso heurístico y general que permite reflexionar en la investigación obtenida, a fin de verificarla para comprender cómo influye en el problema objeto de estudio.

\section{Resultados y discusión}

Tabla 1. Matriz de triangulación.

\begin{tabular}{|c|c|c|c|c|}
\hline Categoría & $\begin{array}{l}\text { Fragmento del } \\
\text { entrevistado }\end{array}$ & Cita del autor & Comentario & Bibliografía \\
\hline $\begin{array}{l}\text { Pensamiento } \\
\text { crítico }\end{array}$ & $\begin{array}{l}\text { Hace referencia a un } \\
\text { proceso cognitivo } \\
\text { de carácter racional, } \\
\text { reflexivo, lógico y } \\
\text { analítico, orientado } \\
\text { al cuestionamiento } \\
\text { sistémico de la realidad } \\
\text { del mundo (A. Orrego, } \\
\text { comunicación personal } \\
\text { de } 15 \text {, agosto de } \\
2019 \text { y S. Ramírez, } \\
\text { comunicación personal, } \\
20 \text { de agosto de 2019). }\end{array}$ & $\begin{array}{l}\text { (Franco et } \\
\text { al., 2018), el } \\
\text { pensamiento } \\
\text { crítico presupone } \\
\text { el conocimiento } \\
\text { de las estructuras } \\
\text { más básicas del } \\
\text { pensamiento" (p. 7). }\end{array}$ & $\begin{array}{l}\text { El pensamiento crítico } \\
\text { moviliza el acto de } \\
\text { entender y establecer } \\
\text { una organización } \\
\text { sistémica de las } \\
\text { ideas, procesos que } \\
\text { promueven en el } \\
\text { estudiante habilidades y } \\
\text { capacidades cognitivas } \\
\text { de análisis, indagación, } \\
\text { reflexión, investigación, } \\
\text { conceptualización y } \\
\text { verificación de datos. }\end{array}$ & $\begin{array}{l}\text { Franco et al. (2018). } \\
\text { Producción investigativa } \\
\text { del pensamiento } \\
\text { reflexivo y crítico } \\
\text { del profesional de } \\
\text { enfermería en el Ecuador. } \\
\text { Revista Publicando, } \\
\text { 5(15), 451-464. } \\
\text { Recuperado de https:// } \\
\text { revistapublicando. } \\
\text { org/revista/index. } \\
\text { php/crv/article/ } \\
\text { download/1352/1358 }\end{array}$ \\
\hline $\begin{array}{l}\text { Estrategia } \\
\text { educativa y } \\
\text { métodos de } \\
\text { enseñanza }\end{array}$ & $\begin{array}{l}\text { El diálogo y diferentes } \\
\text { técnicas interactivas que } \\
\text { permiten la participación } \\
\text { de todas de forma } \\
\text { activa y dinámica (M. } \\
\text { Restrepo, comunicación } \\
\text { personal, } 27 \text { de } \\
\text { septiembre de 2019). } \\
\text { Métodos que conlleven a } \\
\text { la investigación favorecen } \\
\text { el análisis, el discurso } \\
\text { y la objetividad (J. } \\
\text { Jaramillo, comunicación } \\
\text { personal, } 9 \text { de agosto de } \\
\text { 2019). }\end{array}$ & $\begin{array}{l}\text { Cusihualpa (2016): } \\
\text { "se puede indicar } \\
\text { que las estrategias } \\
\text { de aprendizaje son } \\
\text { tareas realizadas } \\
\text { consciente e } \\
\text { intencionalmente, } \\
\text { con el fin de lograr } \\
\text { aprendizajes, en } \\
\text { otras palabras, } \\
\text { son actividades } \\
\text { potencialmente } \\
\text { racionales dado que } \\
\text { son conscientes y } \\
\text { controlables" (p. } \\
\text { 20). }\end{array}$ & $\begin{array}{l}\text { Tanto los métodos } \\
\text { como las estrategias } \\
\text { educativas, } \\
\text { implementadas de } \\
\text { manera activa y } \\
\text { coherente con el } \\
\text { contexto, generan } \\
\text { en los estudiantes } \\
\text { competencias para la } \\
\text { vida. }\end{array}$ & $\begin{array}{l}\text { Cusihualpa (2016). } \\
\text { Estrategias de aprendizaje } \\
\text { en la comprensión lectora } \\
\text { en estudiantes de sexto } \\
\text { ciclo en una institución } \\
\text { educativa. San Isidro } \\
(49-75)\end{array}$ \\
\hline
\end{tabular}




\begin{tabular}{|c|c|c|c|c|}
\hline Categoría & $\begin{array}{l}\text { Fragmento del } \\
\text { entrevistado }\end{array}$ & Cita del autor & Comentario & Bibliografía \\
\hline Docente & $\begin{array}{l}\text { Promueve la reflexión, } \\
\text { la participación y la } \\
\text { construcción conjunta } \\
\text { del conocimiento (L. } \\
\text { Arcila, comunicación } \\
\text { personal del } 5 \text { de agosto } \\
\text { de 2019) } \\
\text { (C. Nayibe, } \\
\text { comunicación personal, } \\
\text { 27, septiembre, 2019). } \\
\text { Incluir diferentes } \\
\text { estrategias y métodos } \\
\text { a los docentes en } \\
\text { formación para su } \\
\text { desarrollo. (A. Cardona, } \\
\text { comunicación personal, } \\
2 \text { de septiembre de } \\
\text { 2019). }\end{array}$ & $\begin{array}{l}\text { Sánchez y González } \\
\text { (2016) “formar } \\
\text { a los educandos } \\
\text { en un ámbito de } \\
\text { interpretación y } \\
\text { comprensión de la } \\
\text { realidad educativa } \\
\text { nacional y regional, } \\
\text { además de estudiar } \\
\text { las implicaciones } \\
\text { sociales, culturales, } \\
\text { cognitivas, } \\
\text { personales y } \\
\text { disciplinares de la } \\
\text { educación desde sus } \\
\text { distintos niveles y } \\
\text { desarrollo" (p. 72). }\end{array}$ & $\begin{array}{l}\text { La importancia del } \\
\text { docente de educación } \\
\text { superior en continuo } \\
\text { crecimiento académico, } \\
\text { que dé cuenta de } \\
\text { las competencias } \\
\text { actitudinales, } \\
\text { conceptuales y } \\
\text { procedimentales de su } \\
\text { praxis pedagógica. }\end{array}$ & $\begin{array}{l}\text { Sánchez y González. } \\
\text { (2016). Saber } \\
\text { pedagógico: fundamento } \\
\text { del espacio docente.edu. } \\
\text { educ, } 19 \text {, p. 241-253. }\end{array}$ \\
\hline
\end{tabular}

Fuente: Elaboración propia

\section{Desarrollo del pensamiento crítico: apreciaciones de los docentes}

Teniendo en cuenta los aportes de los docentes sobre el desarrollo del pensamiento crítico, estos lo entienden como la capacidad de ir más allá de la lectura, lo que implica comprenderlo, interpretarlo y así tener una postura más clara para llevarlo a la práctica en la vida cotidiana. Franco et al. (2018) lo plantean como una modalidad de razonar que moviliza el acto de entender y establecer una organización sistémica de las ideas, procesos que promueven en el estudiante habilidades y capacidades cognitivas de análisis, indagación, reflexión, investigación, conceptualización y verificación de datos. Los participantes aluden a este concepto "como un proceso cognitivo de orden, reflexivo, analítico, deductivo, encaminado al cuestionamiento consecuente de la realidad y del mundo" (A. Orrego, comunicación personal, 15 de agosto de 2019 y S. Ramírez, comunicación personal, 20 de agosto de 2019); esto incita al individuo a pensar, a decodificar su praxis desde la reflexión y bajo una postura objetiva que le aporte en la resolución de problemáticas cotidianas, en la toma de decisiones e iniciativas propias para proponer soluciones asertivas.

De lo anterior se pueden inferir las apreciaciones de los docentes sobre el desarrollo del pensamiento crítico y cómo estas se encuentran relacionadas con la capacidad de "reflexionar, analizar, evaluar, interpretar y proponer ideas frente a diferentes situaciones que le presenta el mundo en el cual habita.” (D. López, comunicación personal, 5 de septiembre de 2019); estas concepciones coinciden con el trabajo investigativo realizado por Facione (2007), al dar como resultado que el pensamiento crítico se centra en la "interpretación, análisis, evaluación, inferencia, explicación y autorregulación” (p. 15). 
De igual modo:

\begin{abstract}
El pensamiento crítico hace referencia a potencializar en el estudiante la capacidad para pensar, evaluar, analizar y criticar de manera constructiva los planteamientos que se realizan en el aula con el fin de proponer nuevas ideas, orientadas a fortalecer la construcción individual y colectiva del conocimiento. (L. Arcila, comunicación personal, 5 de agosto de 2019)
\end{abstract}

Por otra parte, J. Jaramillo comenta que "el desarrollo del pensamiento crítico permite a los estudiantes ser proactivos de su propio conocimiento con una actitud crítica, reflexiva y participativa” (J. Jaramillo, comunicación personal, 9 de agosto de 2019); en este orden de ideas el participante C. Bustamante aporta: "Los estudiantes disfrutan más de actividades donde ellos tengan interacción y participación activa en el proceso de aprendizaje” (C. Bustamante, comunicación personal, 12 de agosto de 2019); ante esto, el acto pedagógico debe garantizar un proceso acorde a los intereses, necesidades y expectativas del contexto educativo y de los estudiantes.

Entonces, la definición de pensamiento crítico no se encuentra muy alejada de las concepciones que tienen los docentes entrevistados y las investigaciones encontradas, las cuales coinciden además con autores como Freire (como se citó en Meza, 2019): "El proceso educativo tiene que fundamentarse en la participación, la autorreflexión y el pensamiento crítico de quienes son sujetos del proceso de enseñanza-aprendizaje" (p. 3); en suma, "los maestros tienen la responsabilidad de generar espacios en las escuelas que les permitan a sus estudiantes desarrollar estrategias para comunicarse asertivamente y fomentar su pensamiento crítico" (Chaux como se citó en Palacio et al., 2019, p. 40). Con este fragmento se infiere que el desarrollo del pensamiento crítico debe estar presente como competencia genérica en todos los procesos de formación, debido a que aprender no solo es memorizar un contenido, sino que se requiere ir más allá para lograr entenderlos y comprenderos, de tal manera que se les pueda dar soluciones diversas y coherentes.

La investigación ha demostrado que los criterios de Paul y Elder (2005), Facione (2007), Franco et al. (2018), Freire (2006) y otros especialistas, consideran el concepto del pensamiento crítico como un asunto de orden analítico, explicativo, complejo del pensar que necesita de la razón para la formación de conocimientos integradores y reflexivos en el sujeto.

\title{
Estrategias educativas y métodos de enseñanza que emergen en el discurso del docente
}

Las apreciaciones de los docentes son relevantes en cuanto son ellos quienes motivan y posibilitan espacios y material educativo para fortalecer el desarrollo del pensamiento crítico 
a través de la lectura, y de todas aquellas actividades donde interactúan los estudiantes y que se desarrollan en los diferentes contextos, de tal manera que sean sujetos con capacidad crítica, autónoma y reflexiva, que permita en ellos la relación con su entorno. Según Meek (2004) leer, por tanto, "es el proceso de convertir el lenguaje escrito en significado. Cuando leemos esperamos descubrir lo que el escritor quiere comunicarnos" (p. 59). Por consiguiente, Méndez (2015) afirma que,

Es necesario llamar de nuevo la atención sobre la importancia de la lectura en la formación de los seres humanos en un mundo donde la información constituye el eje central de las dinámicas que hacen funcionar ámbitos como la política, la ciencia, la administración, las comunicaciones, entre otras. (p. 33)

Por ello, cada documento que el docente sugiera para ser leído debe analizarse; el maestro ha de implementar estrategias didácticas para que sus estudiantes adquieran habilidades lectoras y críticas de lo que se está leyendo, que no se conformen con la lectura literal de un texto, sino que avancen hacia la lectura inferencial y crítica del mismo.

De acuerdo con lo expresado por los participantes en la investigación, se infieren las siguientes apreciaciones:
Son fundamentales los procesos de lectura en todas las asignaturas porque son los que nos van a ayudar en la comprensión de la temática y al mismo tiempo ir adquiriendo un pensamiento propio frente al conocimiento que es bastante amplio, además, papel fundamental para contribuir en el proceso de aprendizaje desde la interlocución con el otro y el análisis de información. (C. Toro, comunicación personal, 17 de septiembre de 2019)

Otros de los participantes manifiestan que "el papel de la lectura permite un proceso de aprendizaje constructivo al tiempo que promueve la reflexión, participación y construcción conjunta del conocimiento, favorece la comprensión y desarrolla procesos cognitivos" (M. Restrepo, comunicación personal, 27, septiembre, 2019). Al respecto, Vásquez (2015) hace énfasis en la capacidad que debe desarrollar un lector, con relación a "elegir o seleccionar sus lecturas, tener un punto de vista manifestado en una opinión, y seguir cultivándose a lo largo de toda la vida” (p. 61). Los teóricos referenciados reconocen que se debe suscitar la lectura en las instituciones de educación superior como una opción para potenciar el desarrollo del pensamiento crítico, al integrar el sentir el pensar y el actuar en los contextos donde se está inmerso.

Además, Torra et al. (2012) expresan que la lectura se debe dar mediante una actitud positiva y motivadora para el desarrollo del pensamiento crítico en los estudiantes, siempre y cuando 
se tenga en cuenta que no hay prescripciones universales válidas al respecto. Sin embargo, existen orientaciones fundamentales para tener en cuenta, resaltando la importancia de las intervenciones del docente como ejercicio pertinente que responde a los intereses y necesidades de los estudiantes, y además ejerza un rol de guía en los procesos de enseñanza y aprendizaje que fomente experiencias enriquecedoras y significativas para el desarrollo integral del sujeto.

En este orden de ideas, los docentes se encuentran permeados por la experiencia que cada uno tiene desde su quehacer pedagógico, es decir, todas aquellas actividades que emplean en los contextos educativos donde interactúan para promover proceso de formación académica mediante estrategias educativas y los métodos de enseñanza utilizados en el aula de clase. Es evidente entonces la necesidad de que estas herramientas sean coherentes a los temas trabajados y sean punto de partida para ir adentrando al estudiante en la práctica de actividades en las que se efectúe el progresar de lo global a lo particular, de lo simple a lo complejo, para contribuir al desarrollo integral de los estudiantes. Díaz (2000) expone: "Son procedimientos que el alumno adquiere y emplea de forma intencional como instrumento flexible para aprender significativamente y solucionar problemas y demandas académicas” (p. 34).

En este sentido, un participante manifiesta "que la interacción entre docente-estudiantes se da a través de conversatorios, mesa redonda, técnicas interactivas, a fin de promover el aprendizaje significativo” (R. Triana, comunicación personal, 7 de septiembre de 2019). Al respecto, Metaute, Flórez, Rugeles y Castaño (2018) mencionan la importancia de los métodos de enseñanza dentro de los procesos formativos de los estudiantes, por tanto, se hace necesario recurrir a los elementos que faciliten la inserción de los estudiantes al contexto social. En esta misma medida, Balestra y Gasca (2017) exponen ideas claras acerca del sistema educativo tolerante, en el cual no solo se valore el resultado como un todo del conocimiento, sino que se realice mediante un proceso valorativo y de formación.

Utilizar estrategias educativas supone algo más que el conocimiento y el manejo de instrucciones en la resolución de una actividad determinada; para ello, es necesario reflexionar sobre la manera de enfrentarlas, superando metodologías tradicionales y memorísticas; además es pertinente implementar panel de conocimiento, mapa conceptual, red de ideas, discusiones, talleres de comprensión lectora, foros, disertaciones, inferencias, ante esto un participante aduce que "todas las estrategias pueden ser funcionales en un momento determinado, puesto que permitan la verificación de una adecuada interpretación y análisis de la temática presentada” (A. Restrepo, comunicación personal, 11 de septiembre de 2019).

Al continuar con la importancia de las estrategias y los métodos de enseñanza en los procesos de aprendizaje, Moreno y Velázquez (2017) argumentan que: 
Los cambios sociales y culturales de la actualidad requieren de ciudadanos formados con una mentalidad crítica, abierta y flexible ante los cambios. Enfrentar esos retos requiere de sistemas educativos que destaquen por la aplicación de métodos de enseñanza que conduzca a potenciar las habilidades del pensamiento crítico y la formación integral de los estudiantes. (p. 54)

Según un participante, la incorporación de la TIC y web 4.0 “favorecen y enriquecen el proceso de enseñanza-aprendizaje, por lo que no es posible pensar un desarrollo del pensamiento crítico si acallamos al otro con la castración de sus herramientas" (I. Vallejo, comunicación personal, 3 de septiembre de 2019). Al respecto, Galindo (2015) menciona la importancia de las competencias digitales y mediales para desarrollar en los estudiantes una postura crítica y reflexiva ante lo que encuentran y leen en la web 2.0.

Para concluir, Acuña (2017) enfatiza en el rol del docente que, además de enseñar los contenidos, debe transversalizar el conocimiento, propiciando el aprender a aprender; para ello, se debe comprender qué estrategias educativas implementar para incentivar la participación y el trabajo en equipo, ante este panorama un participante manifiesta: "Sí, es claro que todos los docentes nos debemos comprometer con este proceso para entender que estamos educando para la vida, teniendo en cuenta las nuevas metodologías digitales para fortalecer los procesos lectores crítico reflexivos" (A. Cardona, comunicación personal, 2 de septiembre de 2019).

\section{Resultados}

A partir del análisis de las categorías desarrollo del pensamiento crítico, estrategias educativas y métodos de enseñanzase se obtienen los siguientes resultados:

Las entrevistas presentan una convergencia entre la teoría y la práctica en relación al pensamiento crítico y la utilización de las estrategias educativas y métodos de enseñanza en el aula, evidenciando un manejo de la pedagogía que propicie el desarrollo del pensamiento crítico; a su vez, que con ella se promueve la adquisición de competencias como ajuste a códigos éticos, capacidad para el trabajo interdisciplinario, apropiación de literatura, manejo adecuado de la información, dominio del discurso oral y escrito propio de la disciplina.

En cuanto a la definición del pensamiento crítico, la mayoría de los docentes entrevistados manifestaron que este concepto es interpretado como un proceso cognitivo que le permite al ser humano reflexionar, analizar, evaluar, interpretar y proponer ideas frente a diferentes situaciones que le presenta el mundo en el cual habita.

En relación con la participación de los entrevistados con preguntas acerca de la importancia del desarrollo del pensamiento crítico en los cursos que acompaña, se resalta la importancia 
de formar competencias críticas, reflexivas y propositivas en los estudiantes; por ello, se deben generar dispositivos tanto para aprender a formular preguntas, como para la crítica sobre lo construido y lo aprendido, así como sobre los métodos para obtener el conocimiento y sobre su aplicación.

Por otro lado, las estrategias y métodos de enseñanza que se implementan en el aula contribuyen al desarrollo de competencias, además del método dialógico que favorece la adquisición de competencias referidas a la escucha, la argumentación, la comunicación no verbal, la capacidad crítica y autocrítica, las relaciones interpersonales y el manejo de frustraciones y sentimientos, generando en los estudiantes competencias tales como actitudes, conocimientos, capacidades y habilidades referidas a la naturaleza de los cursos, se contemplan estrategias como debates, lecturas, exposiciones, estudios de caso, análisis documentales y propuestas de intervención en diferentes contextos educativos.

\section{Discusiones}

El pensamiento crítico y su relación con los métodos y estrategias de aprendizaje se considera un tema muy debatido en los contextos tanto universitarios como en la básica primaria y secundaria, permitiendo de esta manera discutir, notar y cimentar el gran aporte a la información obtenida, la cual posee diversas finalidades y niveles, haciendo referencia a los autores o teóricos que lo han trabajado desde diversas posturas, coyunturas y finalidades, sus apreciaciones trascienden en la función docente y se caracterizan por identificar la mejor estrategia a implementar y no improvisar. A este respecto, Acuña (2017) enfatiza en el rol del docente que, además de enseñar los contenidos, debe transversalizar el conocimiento, propiciando el aprender a aprender; para ello se debe comprender qué estrategias didácticas implementar para incentivar la participación y el trabajo en equipo.

En suma, las universidades deben potenciar en los estudiantes el desarrollo del pensamiento crítico al mismo tiempo que tenga en cuenta la responsabilidad que se tiene desde la formación humana y ética, para entender y confrontar individual y colectivamente los diversos problemas de su contexto para contribuir a la transformación de la realidad. Al respecto, Zubiría (2014) aporta:

Sin embargo, la escuela tradicional se tornó obsoleta en las últimas décadas frente a los sensibles cambios sociales, económicos y políticos vividos a nivel mundial. La sociedad se volvió global e interconectada, el mundo se flexibilizó y diversificó, y cada vez de mayor manera tuvo en cuenta al individuo. (p. 2) 
Así entonces, favorecer el pensamiento crítico en la educación superior debe ser un objetivo indispensable de los currículos de formación, por ser menester de los procesos en los que se está inmerso, por la intervención en múltiples escenarios profesionales, por la evolución científica y especializada, por la calidad de la información que se recibe.

Por consiguiente, debido a que el maestro es planificador de los procesos de enseñanzaaprendizaje, debe ser consecuente con su quehacer educativo, no proponer y replicar lo que otros hacen, sino que debe prepararse para asumir su propia práctica de manera clara, adecuando su trabajo a las necesidades e intereses de la población a la que va intervenir, en una constante actualización que le permita reinventar, resignificar y recodificar su labor profesional y, al mismo tiempo, alcanzar la calidad educativa que tanto se reclama en la actualidad. Según Freire (como se citó en Meza, 2009): "El proceso educativo tiene que fundamentarse en la participación, la autorreflexión y el pensamiento crítico de quienes son sujetos del proceso de enseñanza-aprendizaje” (p. 3).

\section{Conclusiones}

El desarrollo del pensamiento crítico está articulado indiscutiblemente a los procesos de lectura, análisis y comprensión de cada texto que se aborde en las diferentes disciplinas, logrando con ello que los estudiantes fortalezcan las habilidades para razonar, indagar discutir y reflexionar sobre acontecimientos que está viviendo nuestra sociedad y así contribuyan a los desafíos y retos que impone el siglo XXI.

La importancia de implementar las estrategias educativas y métodos de enseñanza en otras áreas curriculares, niveles y contextos para dirigir el proceso de enseñanza- aprendizaje que permita el desarrollo del pensamiento crítico. Si bien es cierto, todas las estrategias y los métodos educativos son efectivos, siempre y cuando se den en un momento y un contexto determinado, con objetivos intencionados y claros; así cada docente debe ser creativo en los procesos de enseñanza-aprendizaje para establecer condiciones apropiadas con metodologías en la investigación desde una posición contextualizada. Las estrategias educativas empleadas por los docentes del componente saberes específicos y disciplinares, contribuyen al desarrollo humano, en competencias relacionadas al diálogo, respeto, equidad, justicia, ética, corresponsabilidad y otredad.

Para finalizar, se deben realizar investigaciones en las cuales se obtenga el punto de vista de los estudiantes, por ser ellos parte importante del proceso formativo y didáctico, por lo cual es oportuno manifestar sus palabras con el propósito de partir de acciones que puedan prevenir y superar brechas en los procesos de formación. En síntesis, en cada uno de los momentos de la vida se requiere de la resolución de problemas, es por esto que se ve como una necesidad apremiante el continuar con el desarrollo de un pensamiento crítico, analítico y creativo que fomente la corresponsabilidad de formar personas competentes para una sociedad justa y equitativa. 


\section{Referencias}

Abanades, M. (2016). Nuevo perfil del docente en la educación superior: formación, competencias y emociones. Opción, 32(8), 17-37.

Acuña, J. (2017). Desarrollo del pensamiento crítico y creativo mediante estrategias interconectadas: estrategias de aprendizaje, lectura crítica, y ABP. Gestión, Competitividad e Innovación, 5(2), 145-162. Recuperado de https:/ / pca.edu.co/investigacion/revistas/ index.php/gci/article/view/106/107.

Balestra,A. y Gasca, L. (2017). Pensamiento crítico, conciencia cultural y tecnología: actividades para cursos de español como L2. Revista Comunicación, 26(2), 49-62. Recuperado de https: / /www.scielo.sa.cr/pdf/com/v26n2/1659-3820-com-26-02-49.pdf.

Bisquerra, R. (2004). Metodología de la investigación educativa. Madrid, España: La Muralla.

Díaz, F. (2000). Estrategias docentes para un aprendizaje significativo. Ciudad de México, México: Mc Graw Hill.

Facione, P. A. (2007). Pensamiento Crítico: ¿Qué es y por qué es importante? Recuperado de https: / / comenio.files.wordpress.com/2007/09/pensamiento_critico.pdf.

Franco, J. A., Piedra, K. A. y Cunalema, J. A. (2018). Producción investigativa del pensamiento reflexivo y crítico del profesional de enfermería en el Ecuador. Revista Publicando, 5(15), 451-464. Recuperado de https: / / revistapublicando.org/revista/index.php/crv/article/ download/1352/1358.

Freire, P. (2006). Pedagogía de la autonomía. México: Siglo XXI Editores.

Galindo, M. A. (2015). Lectura crítica hipertextual en la web 2.0. Actualidades Investigativas en Educación, 15(1), 1-29. doi: dx.doi.org/10.15517/aie. v15i1.16972.

Martínez, J. (2011) Métodos de investigación cualitativa. Research Journal Silogismo, [S.l.], 1,No08.http: / /www.cide.edu.co/ojs/index.php/silogismo/article/view / 64/53

Méndez, J. C. (2015). Sobre la lectura crítica en la Católica del Norte Fundación Universitaria: conceptos, ejemplos y reflexiones. Revista Reflexiones y Saberes, (2), 32-41. Recuperado de http://revistavirtual.ucn.edu.co/index.php/RevistaRyS/article/view/599/1135.

Meek, M. (2004). En torno a la cultura escrita. Ciudad de México, México: Fondo de Cultura Económica.

Metaute, P. M., Flórez, G. A., Rúgeles, P. A. y Castaño, D. A. (2018). La dinamización de las estrategias pedagógicas actuales: una necesidad aplicable a los procesos de enseñanza y aprendizaje de los estudiantes de ingeniería del siglo XXI. Revista Lasallista de Investigación, 15(1), 46-56. Recuperado de http://repository.lasallista.edu.co:8080/ojs/index.php/ rldi/issue/view/82. 
Meza, L. G. (2009). Elementos de pensamiento crítico en Paulo Freire: implicaciones para la educación superior. Revista Digital Matemáticas, 10(1), 45-67. Recuperado de http:// funes.uniandes.edu.co/8073/1/Meza2009Elementos.pdf.

Montes de Oca, N. y Machado, E. F. (2011). Estrategias docentes y métodos de enseñanzaaprendizaje en la Educación Superior. Humanidades Médicas, 11(3), 475-488. Recuperado de http://scielo.sld.cu/scielo.php?script=sci_arttext\&pid=S1727$81202011000300005 \& \operatorname{lng}=$ es\&tlng=es.

Montoya, J. I. y Monsalve, J. C. (2008). Estrategias didácticas para fomentar el pensamiento crítico en el aula. RevistaVirtual Universidad Católica del Norte, (25).

Moreno, W. y Velázquez, M. (2017). Estrategia Didáctica para Desarrollar el Pensamiento Crítico REICE. Revista Iberoamericana sobre Calidad, Eficacia y Cambio en Educación, 15(2), 53-73.

Osses, S. y Jaramillo, S. (2008). Metacognición: un camino para aprender a aprender. Estudios Pedagógicos, 34(1), 187-197. doi: https://dx.doi.org/10.4067/S071807052008000100011.

Palacio, C., Rodríguez, L. J. y Gallego, A. M. (2019). Sentimientos y pensamientos de jóvenes frente al acoso escolar: el suicidio como una alternativa. Revista Eleuthera, 21, 34-47. doi: 10.17151/eleu.2019.21.3.

Paul, R. y Elder, L. (2005). Una Guía Para los Educadores en los Estándares de Competencia para el Pensamiento Crítico. Estándares, Principios, Desempeño Indicadores y Resultados Con una Rúbrica Maestra en el Pensamiento Crítico. Fundación para el Pensamiento Crítico. Recuperado de https: / / www.criticalthinking.org/resources/PDF/SP-Comp_Standards.pdf.

PISA. (2016). Resumen Ejecutivo Colombia en PISA 2015. Recuperado de http: / / virtual.funlam. edu.co $/ \mathrm{mod} /$ lesson $/$ view.php?id=181800\&pageid=62806\&startlastseen=yes .

Romero, C., Villareal, S., Samper, J. y Ospino, I. (2017). Fortalecimiento de las competencias ciudadanas a partir de la lectura crítica en escenarios virtuales. Revista Virtual Universidad Católica del Norte, (51), 216-232. Recuperado de http: / / revistavirtual.ucn.edu.co/index. $\mathrm{php} /$ RevistaUCN/article/view/853/1371

Sampieri, R. H. (2004). Metodología de la investigación. Ciudad de México, México: McGraw-Hill.

Sánchez, T., \& González, S. (2016). Saber pedagógico: fundamento del ejercicio docente. Educ. Educ, 19(2016), 241-253. https://doi.org/10.5294/edu.2016.19.2.4

Steffens, E., Ojeda, J., Decired del C., Martínez, O. M., García, J. E., Hernández, H. G. y Marín, F. V. (2017). Niveles de pensamiento crítico en estudiantes de Universidades en Barranquilla (Colombia). Espacios, 38(30). Recuperado de https://www.revistaespacios. com/a17v38n30/a17v38n30p05.pdf. 
Apreciación del docente para contribuir al desarrollo del pensamiento crítico

Torra, I., Manuel de Villena, I. C., Pérez, M. J., Pagès, T., Valderrama, E., Márquez, M. D. y Triadó, X. (2012). Identificación de competencias docentes que orienten el desarrollo de planes de formación dirigidos a profesorado universitario. Revista de Docencia Universitaria, 10(2), 21-56. Recuperado de https://doi.org/10.4995/redu.2012.6096.

Vásquez, F. (2015). Repensar la lectura y la lectura crítica. Recuperado de http://santillana. com.co/rutamaestra/revistas_pdf/ruta_maestra_v_0010.pdf.

Zubiría, J. (2014). No basta tener buenos docentes. Semana. Recuperado de http:// www.semana.com/educacion/articulo/para-la-excelencia-educativa-no-solo-senecesitanmejores-maestros/382193-3enseñanza-aprendizaje 